\title{
A!
}

This is an electronic reprint of the original article.

This reprint may differ from the original in pagination and typographic detail.

Al-Azawi, Anas; Latikka, Mika; Jokinen, Ville; Franssila, Sami; Ras, Robin H.A.

\section{Friction and Wetting Transitions of Magnetic Droplets on Micropillared Superhydrophobic Surfaces}

Published in:

Small

DOI:

10.1002/smll.201700860

Published: 11/10/2017

Document Version

Peer reviewed version

Please cite the original version:

Al-Azawi, A., Latikka, M., Jokinen, V., Franssila, S., \& Ras, R. H. A. (2017). Friction and Wetting Transitions of Magnetic Droplets on Micropillared Superhydrophobic Surfaces. Small, 13(38), 1-7. [1700860].

https://doi.org/10.1002/smll.201700860

This material is protected by copyright and other intellectual property rights, and duplication or sale of all or part of any of the repository collections is not permitted, except that material may be duplicated by you for your research use or educational purposes in electronic or print form. You must obtain permission for any other use. Electronic or print copies may not be offered, whether for sale or otherwise to anyone who is not an authorised user. 
DOI: 10.1002/ ((please add manuscript number))

Article type: ((Full Paper))

\title{
Friction and Wetting Transitions of Magnetic Droplets on Micropillared Superhydrophobic Surfaces
}

Anas Al-Azawi, Mika Latikka, Ville Jokinen, Sami Franssila, and Robin H. A. Ras*

\author{
A. Al-Azawi, M. Latikka, Prof. R. H. A. Ras \\ Department of Applied Physics, Aalto University \\ P.O. Box 15100, FI-00076 Aalto, Espoo, Finland \\ Dr. V. Jokinen, Prof. S. Franssila \\ Department of Material Science and Engineering, Aalto University \\ P.O. Box 13500, FI-00076 Aalto, Espoo, Finland \\ E-mail: robin.ras@aalto.fi
}

Keywords: superhydrophobicity, droplet oscillations, friction, wetting transition

\begin{abstract}
Reliable characterization of wetting properties is essential for the development and optimization of superhydrophobic surfaces. Here we study the dynamics of superhydrophobicity including droplet friction and wetting transitions by using droplet oscillations on micropillared surfaces. Analyzing droplet oscillations by high-speed camera makes it possible to obtain energy dissipation parameters such as contact angle hysteresis force and viscous damping coefficients, which indicate pinning and viscous losses, respectively. We show that the dissipative forces increase with increasing solid fraction and magnetic force. For $10 \mu \mathrm{m}$ diameter pillars, the solid fraction range within which droplet oscillations are possible is between $0.97 \%$ and $2.18 \%$. Beyond the upper limit, the oscillations become heavily damped due to high friction force. Below the lower limit, the droplet is no longer supported by the pillar tops and undergoes a Cassie-Wenzel transition. This transition is found to occur at lower pressure for a moving droplet than for a static droplet. Our findings can help to optimize micropillared surfaces for low-friction droplet transport.
\end{abstract}




\section{Introduction}

The non-wettability of microstructured superhydrophobic surfaces offers remarkable properties such as self-cleaning, ${ }^{[1-4]}$ drag reduction,,${ }^{[5-9]}$ anti-fouling, ${ }^{[10]}$ and icing resistance $^{[11-15]}$ and have gained substantial attention because of their application in surface modification ${ }^{[16,17]}$ and micro/nanofluidics. ${ }^{[18,19]}$ It is well understood that superhydrophobicity is attributed to regular or irregular surface topographies combined with chemical compositions that minimize surface energy. ${ }^{[20-23]}$ Water droplets on patterned surfaces are known to exist typically in one of two possible wetting states. ${ }^{[24]}$ In the Wenzel state the droplet is impaled in the surface topography fully wetting the underlying surface resulting in limited droplet mobility. In the Cassie-Baxter state air remains trapped beneath the droplet and only the top parts of the surface features are wetted, providing low droplet friction and high mobility.

Transition between these two wetting modes has been studied through experiments, ${ }^{[25-32]}$ analytical models ${ }^{[33,34]}$ and simulations. ${ }^{[35,36]}$ So far investigation of Cassie-Wenzel transition (CW transition) has concentrated on static droplets that undergo transition to Wenzel state by mechanical compression, ${ }^{[24]}$ magnetic force, ${ }^{[30]}$ electrostatic force, ${ }^{[27]}$ evaporation, ${ }^{[26]}$ gravity ${ }^{[31]}$ or by vibration. ${ }^{[32]}$

Assuming that Wenzel state is a global energy minimum for a given system, the transition is irreversible unless external force is applied to overcome the energy barrier for a reverse transition. ${ }^{[30]}$ This can be facilitated by implementing a superhydrophobic surface with dual-scale topography. ${ }^{[29]}$

Usually the wetting properties of surfaces are determined by measuring the contact angle (CA) of a water droplet on the surface. The CA depends on surface energies, substrate topography and the dynamic state of the contact line. There is usually a difference between the angle observed at the advancing edge of the droplet as it moves or its volume 


\section{WILEY-VCH}

increases (the advancing contact angle, ACA) and at the receding edge or when its volume is decreased (the receding contact angle, RCA). The difference between the advancing and receding contact angle is termed the contact angle hysteresis $(\mathrm{CAH})$, which gives a measure of surface pinning. The larger the hysteresis value is, the more the droplet will be pinned on the surface. Surfaces with low hysteresis value are usually desired as this means that droplets will roll off easily enabling multiple types of applications.

The typical approach for characterizing superhydrophobic surfaces relies heavily on optical goniometers. ${ }^{[37]}$ Despite the fact that $\mathrm{CA}$ and $\mathrm{CAH}$ can be evaluated by a goniometer, pinpointing the contact line exactly at the interface between the droplet and the surface is difficult for very high CA values. ${ }^{[37-39]}$ The reliability of the CA measurements is thus limited by optical errors. To overcome the limitations of CA method, we had developed a method based on freely decaying oscillations of magnetic water droplets to quantify energy dissipation on superhydrophobic surfaces. ${ }^{[40]}$ The method allows simultaneous evaluation of both contact line friction and viscous damping forces. So far, droplet oscillations have not been systematically studied on well-defined surface topographies.

In this work, friction dependency on the solid fraction of the surface structure is quantified by conducting transverse droplet oscillations on superhydrophobic surfaces under controlled lateral and normal forces. In addition, we investigate the robustness of metastable Cassie-Baxter state using both static and laterally moving droplets. The effect of lateral droplet motion on the $\mathrm{CW}$ transition has not been previously studied to our knowledge. The magnetic droplet is dispensed on a micropillared superhydrophobic surface, which is located above a permanent magnet creating a magnetic potential well for the droplet. The magnetic droplet is driven out of equilibrium by quickly moving the magnet horizontally. The droplet follows due to magnetic force and starts to oscillate around the magnet axis when the magnet is stopped. These oscillations are captured with a 


\section{WILEY-VCH}

high-speed camera and analyzed to measure viscous dissipation as well as friction of the moving droplet.

The influence of the solid fraction is investigated by using micropillared surfaces with different pillar separations. For each surface oscillations are repeated at various distances above the magnet, which enables to vary the forces involved. This way, the robustness of Cassie state is evaluated while parameters regarding friction and dissipation are extracted from each captured oscillation.

\section{Results and Discussion}

\subsection{Droplet oscillations on micropillared surfaces}

Four micropillared superhydrophobic surfaces that share the same geometrical pattern but each has a distinct value of solid fraction were achieved by varying the structure pitch. Two series of experiments using a concentrated and a dilute ferrofluid with nanoparticle concentration equal to 4 vol- $\%$ and 0.2 vol- $\%$, respectively, are performed. Droplet oscillations are conducted with increasing normal force at different vertical distances above a permanent magnet. The total potential energy of a magnetic droplet above the magnet is $\boldsymbol{U}_{\text {total }}=-\boldsymbol{\mu}_{\mathbf{0}} \boldsymbol{H} \boldsymbol{M V}+\boldsymbol{m g} \boldsymbol{z}$, where $M, V, m$ and $z$ denote the droplet magnetization, volume, mass and distance above the magnet. $H$ is the magnetic field experienced by the droplet, which can be approximated as parabolic near the magnet axis, $\boldsymbol{H}=\boldsymbol{H}_{\mathbf{0}}-\boldsymbol{c} \boldsymbol{x}^{2} / \mathbf{2}$, where $H_{0}$ is the field strength on the axis, $c$ is the curvature of the field and $x$ is the distance from the center of the potential well created by the permanent magnet. The field strength and curvature are considered non-variable because the same magnet is used throughout all the experiments. The magnetic potential energy of the droplet increases when the droplet is displaced from the symmetry axis of the magnetic 


\section{WILEY-VCH}

potential well as the magnet is laterally moved. Therefore, the droplet will experience a Hookean restoring force $\boldsymbol{F}_{\text {ext }}=\frac{-d U_{\text {total }}}{d x}=-\mu_{0} V \boldsymbol{c x}\left(\boldsymbol{M}+\boldsymbol{H} \frac{d M}{d \boldsymbol{H}}\right)=-\boldsymbol{k x}$, where $\boldsymbol{k}=$ $\boldsymbol{\mu}_{\mathbf{0}} \boldsymbol{V} \boldsymbol{c}\left(\boldsymbol{M}+\boldsymbol{H} \frac{d \boldsymbol{M}}{d \boldsymbol{H}}\right)$ which brings the droplet into oscillatory motion that is damped by dissipative forces, namely friction forces and viscous damping (Figure 1a, Movie S1). In order to determine the exact contribution from these forces, the harmonic oscillations are analyzed by tracking the droplet position with time and fitting the data with the solution of the general harmonic oscillator.

The downward normal force $\boldsymbol{F}_{\boldsymbol{N}}=\frac{-\boldsymbol{d} U_{\text {total }}}{\boldsymbol{d z}}=-\boldsymbol{m} \boldsymbol{g}_{E}$, where $\boldsymbol{g}_{E}=-\boldsymbol{\mu}_{\mathbf{0}} \boldsymbol{V} \frac{\boldsymbol{d H}}{\boldsymbol{d z}}\left(\boldsymbol{M}+\boldsymbol{H} \frac{\boldsymbol{d} \boldsymbol{M}}{\boldsymbol{d} \boldsymbol{H}}\right)$, acting on the droplet can be increased by bringing the superhydrophobic surface closer to the magnet. Oscillations are repeated with increasing normal force until the superhydrophobic surface reaches a position above the magnet where no oscillation can be performed either because of increased friction or wetting transition (Figure 1b, Figure S1 and Movies S2, S3 and S4).
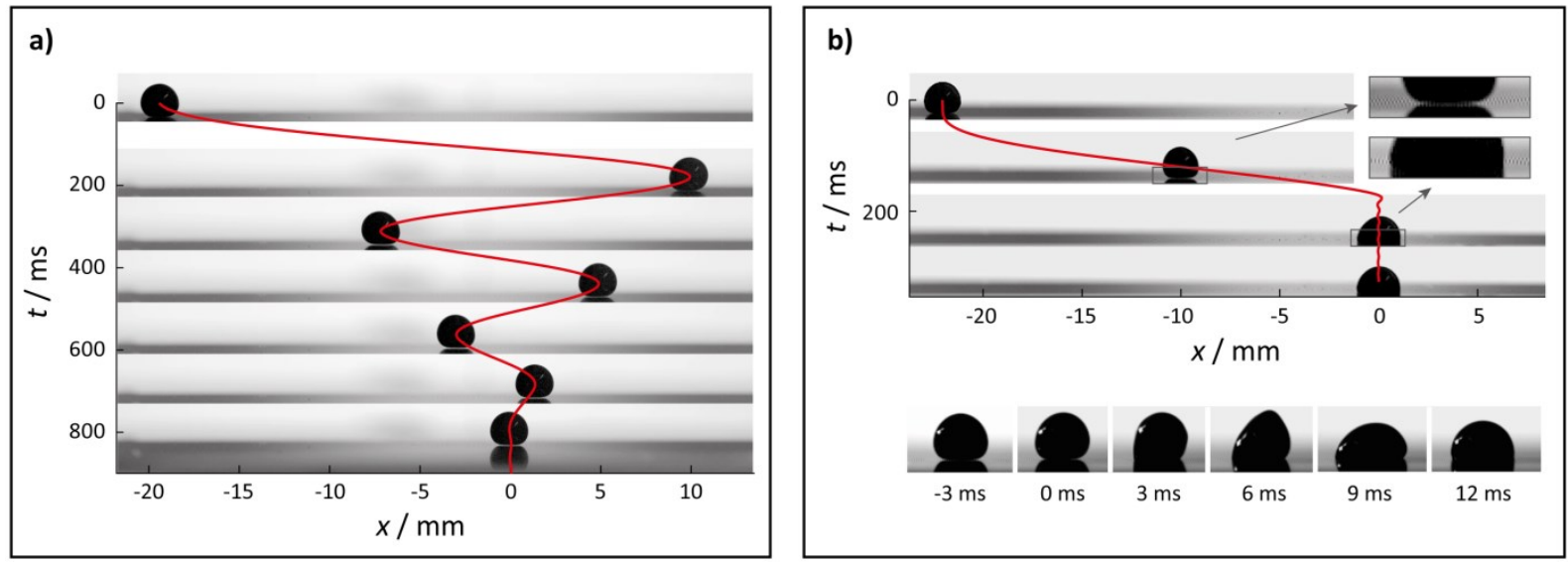

Figure 1. Magnetic droplet oscillation and wetting transition on a micropillared superhydrophobic surface. Droplet oscillation are initiated by the lateral motion of the magnet below the superhydrophobic surface. a) Snapshots showing the extreme points of the damped oscillatory motion. The normal force is $F_{\mathrm{N}}=210 \pm 20 \mu \mathrm{N}$. b) Wetting transition upon lateral motion of the droplet. The 


\section{WILEY-VCH}

\subsection{Dissipative forces}

To investigate the influence of surface solid fraction on the friction and wetting transition, oscillations are performed using equal normal force on four superhydrophobic surfaces with different pillar pitch values. As is evident from Figure 2 and Movies S1, S5, S6 and S7, the damping of oscillations increases with the solid fraction $\phi$.

a)

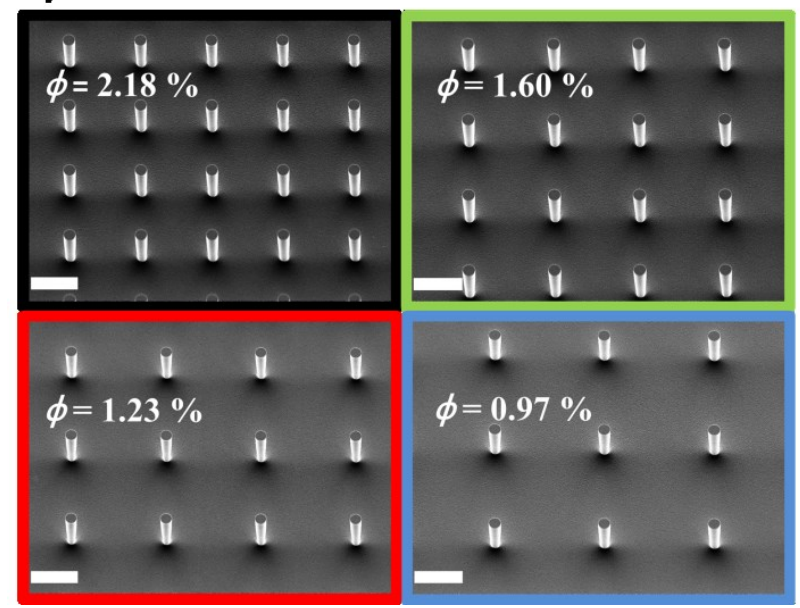

b)
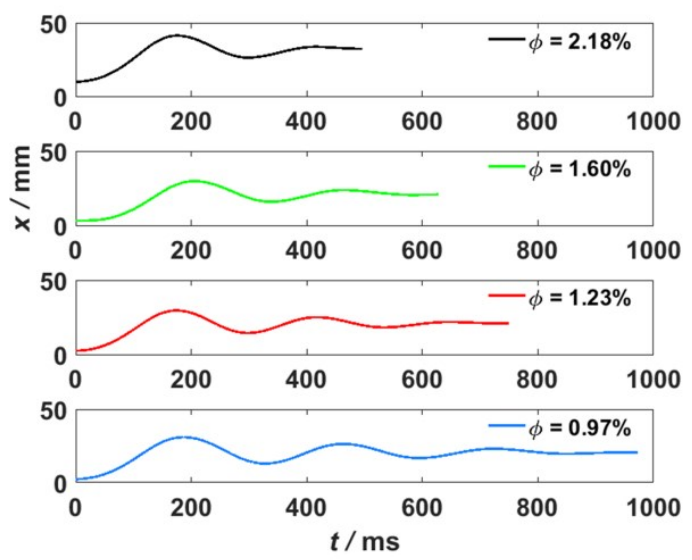

Figure 2. a) Scanning electron microscope images of the micropillars with pillar diameter $=10 \mu \mathrm{m}$ and varying solid fraction $\phi$. Scale bar $40 \mu \mathrm{m}$. b) The influence of the solid fraction $\phi$ on the duration of magnetic droplet oscillation. The end points of the curves correspond to the points where the droplet ceases to move.

To assess the dissipative forces and their dependence on the solid fraction, $\mathrm{CAH}$ force and viscous dissipation coefficients were extracted from concentrated (4 vol-\%) ferrofluid droplet oscillations performed at incrementally decreasing distances above the magnet and plotted against the droplet three-phase contact line length $L$ and the contact area $A$, which in turn increase with the normal force. We use Young-Laplace equation to calculate 


\section{WILEY-VCH}

apparent contact line lengths and apparent contact areas, which correspond to apparent contact between the droplet and the substrate. Microscopically the droplet is only in contact with the pillar tops and thus the real contact area used in the plot is $A=\phi A_{\text {apparent }}$. Similarly, the real contact line length $L=\phi L_{\text {apparent }}$ refers to the contact line on pillar tops at the outer edge of the droplet. Contribution to $\mathrm{CAH}$ force originates from liquid depinning occurring at the edge of droplet contact area.

The position of the droplet $x(t)$ was fitted with the solution of the general harmonic oscillator $\boldsymbol{m}\left(\frac{d^{2} x}{d t^{2}}\right)=-\boldsymbol{k} \boldsymbol{x}-\boldsymbol{F}_{\boldsymbol{\eta}} \pm \boldsymbol{F}_{\boldsymbol{\mu}}$, where $\boldsymbol{F}_{\boldsymbol{\eta}}=\mathbf{2} \boldsymbol{\beta}\left(\frac{d x}{d t}\right)$ is the viscous force and $\boldsymbol{F}_{\boldsymbol{\mu}}=$ $\frac{\boldsymbol{L}}{2} \boldsymbol{\gamma}\left(\boldsymbol{\operatorname { c o s }} \boldsymbol{\theta}_{\boldsymbol{R}}-\cos \boldsymbol{\theta}_{\boldsymbol{A}}\right)$ is the CAH force. $\beta, \gamma, L, \theta_{A}$ and $\theta_{R}$ are viscous damping coefficient, surface tension, three-phase contact line length, ACA and RCA, respectively. As shown in Figure 3a, for each surface the CAH force increases monotonically with the normal force. The length of the three-phase contact line and the contact area increase with increasing normal force, which determines the shape of the droplet. Given the linear dependence between the $\mathrm{CAH}$ force and the length of the three-phase contact line, the increase in normal force translates into increase in $\mathrm{CAH}$ force. The viscous dissipation coefficient also increases with the normal force being linearly dependent with the contact area (Figure 3b). In addition, Figure 3 demonstrates the dependency of both CAH force and viscous dissipation on the solid fraction, enabling to distinguish the obtained dissipative forces from each surface. The oscillation experiments were repeated on the same surfaces but with the dilute ( 0.2 vol-\%) ferrofluid, whose surface tension, density and viscosity are essentially the same as water. ${ }^{[40]}$ The total energy dissipation from droplet oscillations conducted on the four superhydrophobic surfaces of varying solid fraction is presented in Figure 4a. All the oscillations are subjected to the same normal force. The results reveal the same tendency observed in the case of concentrated ferrofluid as the envelope function of the total energy dissipation increases with the solid fraction 


\section{WILEY-VCH}

value. Similarly, the total energy dissipation increases with the normal force, when the solid fraction is kept constant as shown in Figure 4b. Dilute ferrofluid experiences weaker magnetic restoring force due to the lower nanoparticle concentration and therefore less oscillations can be observed. As a result it becomes difficult for the analysis method to distinguish the two dissipative forces when droplets of dilute ferrofluid are used to characterize the micropillared surfaces. Therefore, it is preferred to plot the total energy dissipation instead of plotting both contributions of the energy dissipation separately.

a)

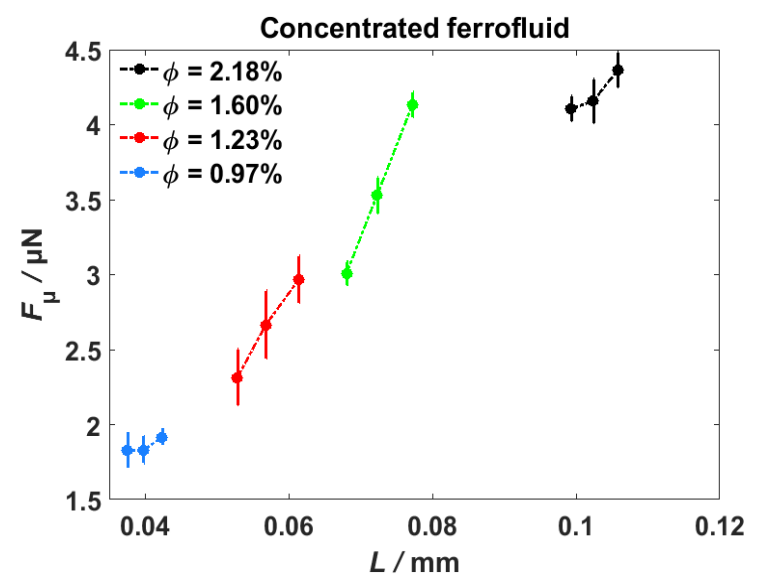

b)

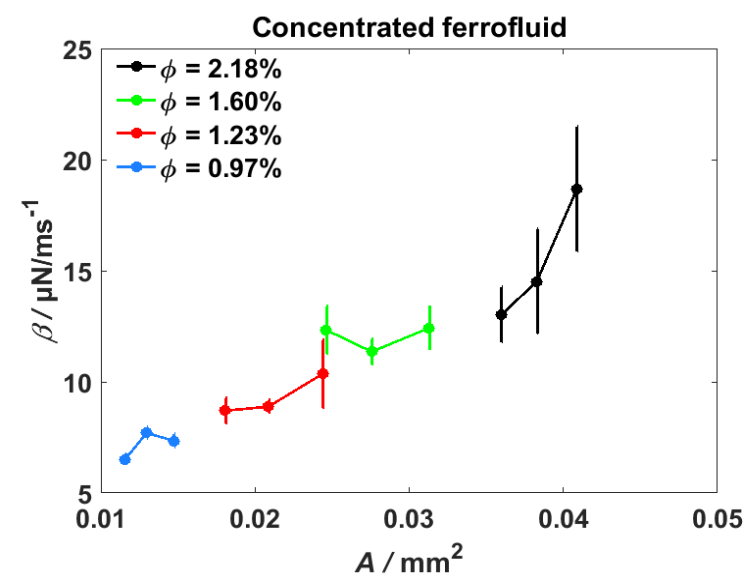

Figure 3. Contact angle hysteresis (CAH) force $F_{\mu}$ and viscous dissipation coefficient $\beta$ as a function of the three-phase contact line length $L$ (a) and contact area $A$ (b), respectively for four superhydrophobic surfaces of varying solid fraction $\phi$.

The advancing contact angle (ACA) and receding contact angle (RCA) on five micropillared superhydrophobic surfaces using the common sessile drop method are presented in 


\section{WILEY-VCH}

Table 1, where each solid fraction value and its corresponding value of CAH are given. The measurements yield lower $\mathrm{CAH}$ as the value of surface solid fraction decreases. The surface with solid fraction value $\phi=3.14 \%$ was excluded from oscillation experiments because the yielded oscillations are too strongly damped for analysis.

Compared to sessile drop method, droplet oscillations can reveal more information on the dynamics of droplet motion under tunable normal force.

a)

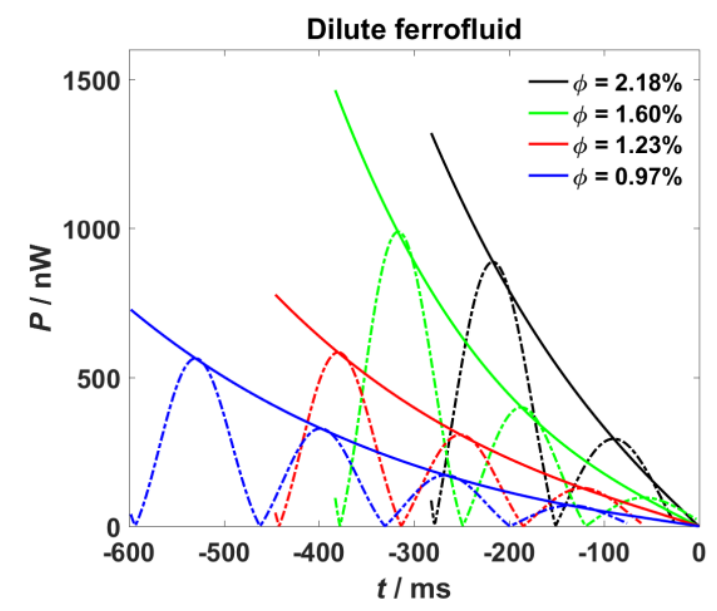

b)

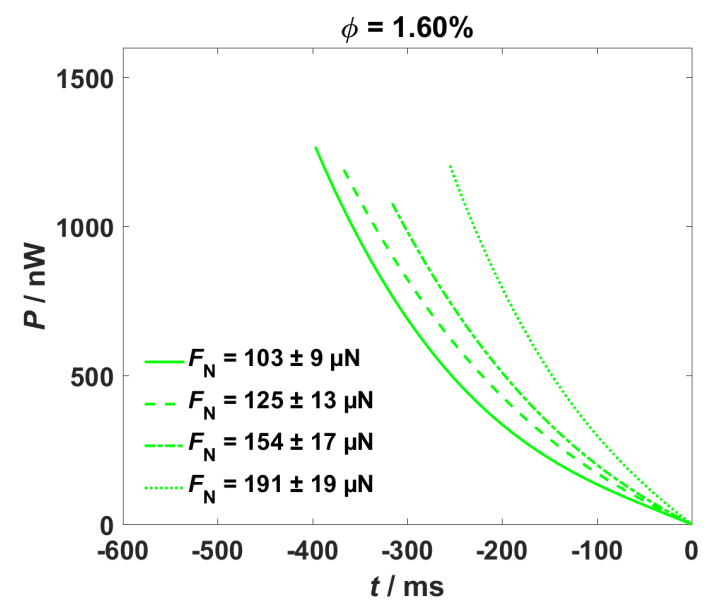

Figure 4. a) The total energy dissipation with the corresponding envelope function of the damped oscillations for four surfaces of varying solid fraction $\phi$ experiencing the same normal force $F_{\mathrm{N}}=113 \pm 11 \mu \mathrm{N}$. b) The envelope function of the total energy dissipation of dilute droplet oscillations on superhydrophobic surface with solid fraction $\phi$ equal to $1.60 \%$. The oscillations are repeated at four different distances above the magnet. The normal force increases from $F_{\mathrm{N}}=103 \pm 9 \mu \mathrm{N}$ at $10 \mathrm{~mm}$ above the magnet to $F_{\mathrm{N}}=191 \pm 19 \mu \mathrm{N}$ at $4 \mathrm{~mm}$ above the magnet. 
Table 1. Advancing contact angle, receding contact angle and contact angle hysteresis of water droplet on micropillared superhydrophobic surfaces of varying solid fraction. The measurements are repeated three times at different spots on the substrates.

\begin{tabular}{|l|l|l|l|}
\hline Solid fraction $\phi$ & ACA $\left(^{\circ}\right)$ & RCA $\left(^{\circ}\right)$ & CAH $\left(^{\circ}\right)$ \\
\hline $3.14 \%$ & $170.3 \pm 0.8$ & $156.8 \pm 1.7$ & $13.5 \pm 1.9$ \\
\hline $2.18 \%$ & $171.4 \pm 1.0$ & $157.2 \pm 1.3$ & $14.2 \pm 1.6$ \\
\hline $1.60 \%$ & $170.2 \pm 0.8$ & $160.0 \pm 0.6$ & $10.2 \pm 1.0$ \\
\hline $1.23 \%$ & $170.5 \pm 0.3$ & $160.6 \pm 2.0$ & $9.9 \pm 2.0$ \\
\hline $0.97 \%$ & $171.5 \pm 1.8$ & $164.3 \pm 1.3$ & $7.2 \pm 2.2$ \\
\hline
\end{tabular}

\subsection{Cassie - Wenzel Transition}

We use the magnetically controllable normal force to determine critical pressure values for static and moving droplets in Cassie-Baxter state for each surface. Critical pressure is defined as the highest pressure that the structure can sustain before $\mathrm{CW}$ transition. Critical pressures for a static droplet is measured by increasing the normal force by bringing the superhydrophobic surface closer to the magnet until the droplet experiences a transition. For a moving droplet the critical pressure is determined similarly, but droplet oscillations are induced for each normal force value.

Just before the transition occurs, the total force applied by the critical pressure $P_{c}$ on the water-air interface at the pillar interspacing in each unit cell area $A_{\mathrm{c}}$ is given as $P_{c}(1-$ 


\section{WILEY-VCH}

ф) $A_{c}$. This force is balanced by the total vertical component of the water-air interfacial tension acting on the pillar perimeter $S$, expressed as $-\gamma S \cos \theta_{\mathrm{A}}$, where $\theta_{\mathrm{A}}=119^{\circ}$ is the measured advancing contact angle of a concentrated ferrofluid droplet on a flat region of the superhydrophobic surface. As a result, the critical pressure can be calculated as $\boldsymbol{P}_{\boldsymbol{c}}=$ $\frac{-\gamma S \cos \theta_{A}}{(1-\varphi) A_{C}}$

When the pressure caused by the normal force exceeds the critical pressure, the solidliquid-air contact line depins from the side of the pillars initiating the transition. The dependency of the experimentally determined CW transition pressure on the solid fraction for the moving and static droplets as well as the analytically calculated critical pressure is shown in Figure 5a and Figure S2. Both analytical and experimental transition pressure values increase as the solid fraction increases from $\phi=0.97 \%$ to $\phi=2.18 \%$. The corresponding experimental transition pressure of a moving droplet increases from $168 \mathrm{~Pa}$ to $330 \mathrm{~Pa}$.

It is noteworthy that the experimental transition pressure of a static droplet is about $76 \mathrm{~Pa}$ higher than the analytically calculated pressure. The difference is attributed to nanotrench roughness of the sidewall (Figure S3) originating from the alternating cycles of deposition and etching during the silicon etching Bosch process. The solid-liquid-air contact line can be pinned on the sidewall structure, which explains the excess pressure required for the transition. Importantly, we observe that transition occurs at lower pressure values for moving droplets compared to static droplets.

As illustrated in Figure 5b, the experimental pressure parameter space is divided into four regions, which are droplet oscillations, heavily damped droplet motion, $\mathrm{CW}$ transition region of dynamic droplet and CW transition region of static droplet. In the droplet oscillations region, the harmonic oscillations are recorded. Below the lower limit of this region, the pressure is too weak to allow oscillations suitable for the analysis. The heavily 


\section{WILEY-VCH}

damped motion region is above the upper limit of the droplet oscillation region. The droplets are in Cassie-Baxter state and mobile but no sufficient oscillatory motion can be generated due to high friction.

a)

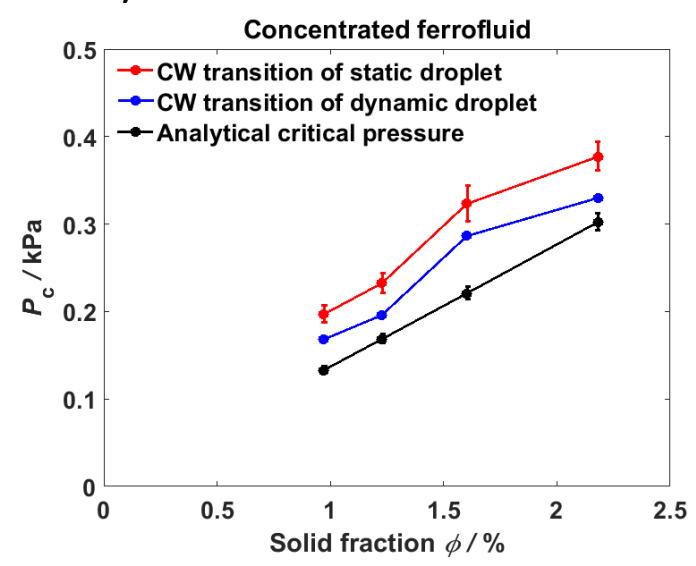

b)

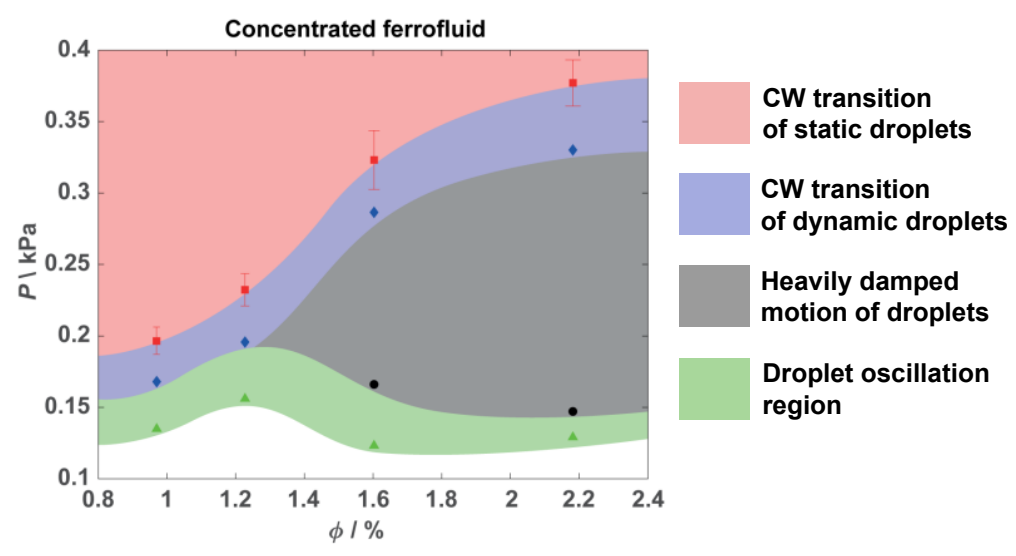

Figure 5. a) Critical pressure $P_{\mathrm{C}}$ as function of solid fraction $\phi$. The experimental transition pressure values are compared with the analytical values for the four superhydrophobic surfaces. The critical pressure for a moving droplet is lower compared to a static droplet. b) Pressure parameter space showing the four regions of droplet movement and wetting transition.

Droplet oscillations can only be performed if the droplet stays in Cassie-Baxter state and the dissipative forces are not too large compared to magnetic restoring force. As expected, the superhydrophobic surface with the highest value of solid fraction $\phi=2.18 \%$ exhibits robustness against $\mathrm{CW}$ transition but also high friction, which leads to narrow pressure interval within which droplet oscillations can be recorded, as shown in the Figure 5b. Droplet oscillations become too damped for analysis as the magnetic field induced pressure increases to $147 \mathrm{~Pa}$ and $166 \mathrm{~Pa}$ for surfaces with $\phi=2.18 \%$ and $\phi=1.60 \%$, respectively. Obtaining CAH force $F_{\mu}$ values beyond that is not feasible as seen in the high friction region. On the other hand, the superhydrophobic surface with the lowest solid fraction $\phi=0.97 \%$ manifests 


\section{WILEY-VCH}

low friction but the oscillations can only be performed on a limited pressure range (135 $\mathrm{Pa}-$ $168 \mathrm{~Pa})$ due to low transition pressure, as seen in Figure $5 \mathrm{~b}$.

\section{Conclusions}

In this work we have used oscillating ferrofluid droplets for characterizing superhydrophobic surfaces and extracted important parameters like contact angle hysteresis force and viscous damping coefficient that cannot be accurately obtained by other methods. The characterization revealed that by decreasing the solid fraction, both viscous damping and friction force are reduced. Moreover, we have compared Cassie-Wenzel transition pressure for a moving droplet with a static droplet. We found out that Cassie-Wenzel transition for a moving droplet occurs more easily than for a static droplet brought near the magnet. The transition pressure of both moving and static droplet increases with increased solid fraction. Various applications of superhydrophobic surfaces require low droplet friction combined with a stable Cassie-state. We have shown in this paper that these requirements can be investigated using field-induced oscillations of magnetic droplets. For a micropillared surface with $10 \mu \mathrm{m}$ diameter pillars, the optimal solid fraction for oscillatory droplet motion is within $0.97 \% \leq \varphi$ $\leq 2.18 \%$ according to our findings.

\section{Experimental section}

\subsection{Ferrofluid synthesis and characterization}

Superparamagnetic iron oxide nanoparticles were synthesized in water using coprecipitation method and stabilized with citric acid near $\mathrm{pH} 7{ }^{[41,42]}$ The resulting ferrofluid was diluted 


\section{WILEY-VCH}

with Milli-Q water to concentrations of 0.2 vol-\% ("dilute ferrofluid") and 4 vol-\%

("concentrated ferrofluid"). Magnetic properties of the ferrofluids (Figure S4a and b) were measured using a vibrating sample magnetometer (VSM, Quantum Design PPMS Dynacool). Approximately $7 \mu 1$ of ferrofluid was placed in standard polypropylene powder cups sealed with vacuum grease and parafilm. Hysteresis loops were measured in temperature of $300 \mathrm{~K}$ using $40 \mathrm{~Hz}$ frequency with $1 \mathrm{~mm}$ peak amplitude in magnetic fields between -9 and $9 \mathrm{~T}$. Similar measurements were performed with an empty powder cup and substracted from ferrofluid measurements to remove background signal from the powder cup. Transmission electron micrographs (Figure S4a and b) of the iron oxide nanoparticles were taken with FEI Tecnai 12 Bio Twin TEM using $120 \mathrm{kV}$ voltage.

\subsection{Fabrication of superhydrophobic surfaces}

Superhydrophobic surfaces were fabricated by implementing bulk micromachining process involving photolithography and silicon etching. First, adhesion promoter hexamethyldisilazane was applied on silicon wafers followed by spin-coating using positive tone resist AZ5214E at $4000 \mathrm{rpm}$ for $30 \mathrm{~s}$ and soft baking for $2 \mathrm{~min}$ at $90{ }^{\circ} \mathrm{C}$, then wafers were exposed through photo mask to UV dose equal to $3.18 \mathrm{~J} \mathrm{~cm}^{-2}$. After exposure, the wafers were submerged into the developer solution AZ351B: $\mathrm{H}_{2} \mathrm{O}(1: 5)$ for 1 min followed by rinsing in deionized (DI) water for $5 \mathrm{~min}$ and hard baked for $3 \mathrm{~min}$ at $120^{\circ} \mathrm{C}$.

The transfer of the patterned photo-resist layer into the underlying silicon substrate was achieved by Bosch etch process. Silicon etching was carried out in advanced silicon etcher (ASE), which is RIE ICP system by Surface Technology Systems (STS). The duration of each cycle is $20 \mathrm{~s}$ (etching: $13 \mathrm{~s}$ and passivating: $7 \mathrm{~s}$ ) and the process gases are $\mathrm{C}_{4} \mathrm{~F}_{8}$ and $\mathrm{SF}_{6}$, for etching cycle only $\mathrm{SF}_{6}$ is deployed. Silicon etching step yielded pillar height equal to $40 \mu \mathrm{m}$ 


\section{WILEY-VCH}

according to the measurement done by stylus profilometer Dektak. After complete etch, the resist layer was stripped by first placing the processed wafers in an acetone bath subjected to ultra sound for $15 \mathrm{~min}$ then moved to a new acetone bath for 5 min followed by cleaning with isopropanol (IPA) and deionized (DI) water for $3 \mathrm{~min}$. and $5 \mathrm{~min}$, respectively. Finally the remaining resist residues were removed by Piranha treatment for $12 \mathrm{~min}$ at $110{ }^{\circ} \mathrm{C}$.

In order to obtain superhydrophobic surface, low surface energy coating was applied to the surface. This was done by vapor deposition of $1 H, 1 H, 2 H, 2 H$-perfluorodecyltrichlorosilane (FDTS) $\mathrm{C}_{10} \mathrm{H}_{4} \mathrm{Cl}_{3} \mathrm{~F}_{17} \mathrm{Si}$. The deposition time and temperature was set to $8 \mathrm{~h}$ and $85{ }^{\circ} \mathrm{C}$ respectively. The deposition was carried out in closed Pyrex petri dish on a hot plate where the structured silicon surface was placed close to FDTS.

\subsection{Magnetic droplet oscillations}

Harmonic magnetic droplet oscillations were investigated using micromanipulator and high speed camera (Phantom Miro 310). The micromanipulator enabled the vertical movement of the surface and controlling the normal force acting on the droplet. In addition, the magnet beneath the surface was moved in lateral direction with a linear stage.

A $5 \mu \mathrm{L}$ magnetic droplet was dispensed on the superhydrophobic surface using a fixed needle (Hamilton 75N Syringe) well above the magnet to avoid droplet collapse to Wenzel state. The magnet was quickly moved in lateral direction by $20 \mathrm{~mm}$ to displace the droplet, producing oscillations that were captured at $1000 \mathrm{fps}$ with the high speed camera. Usually three successive oscillations were recorded before the droplet was removed from the surface to avoid change in droplet mass due to evaporation. The surface was washed with DI water, a 


\section{WILEY-VCH}

new droplet was deposited and three other oscillations were recorded. After that the surface was lowered $2 \mathrm{~mm}$ towards the magnet to perform experiments with increased normal force.

\section{Acknowledgements}

This work was supported by the Academy of Finland (Centres of Excellence Programme (2014-2019) and projects \#256206, \#266820, \#297360 and \#263560). This work made use of the Aalto University Nanomicroscopy Center premises and the cleanroom facilities of Micronova Nanofabrication Center.

\section{Supporting Information}

Supporting Information is available from the Wiley Online Library or from the author.

\section{References}

[1] A. Solga, Z. Cerman, B. F. Striffler, M. Spaeth, W. Barthlott, Bioinsp. Biomim 2007, 2, 126-134.

[2] J. Kong, T. Kim, J. Kim, J. Park, D. Lee, S. Kim, J. Kim, Nanoscale 2014, 6,14531461.

[3] H. Jin, X. Tian, O. Ikkala, R. H. A. Ras, ACS Appl. Mater. Interfaces 2013, 5, 485-488.

[4] Q. Wang, X. Yao, H. Liu, D. Quéré, L. Jiang, Proc. Natl. Acad. Sci. 2015, 112, 92479252.

[5] C. Lee, C. J. Kim, Langmuir 2009, 25, 12812-12818.

[6] J. P. Rothstein, Annu. Rev. Fluid Mech 2010, 42, 89-109.

[7] G. D. Bixler, B. Bhushan, Nanoscale 2014, 6, 76-96. 


\section{WILEY-VCH}

[8] H. Mertaniemi, V. Jokinen, L. Sainiemi, S. Franssila, A. Marmur, O. Ikkala, R. H. A. Ras, Adv. Mater. 2011, 23, 2911-2914.

[9] G. McHale, in Non-Wettable Surfaces, RSC Soft Matter No. 5 (Eds: R. H. A. Ras and A. Marmur), Cambridge, UK 2017, pp. 253-284.

[10] P. Zhang, L. Lin, D. Zang, X. Guo, M. Liu, Small 2016, DOI 10.1002/smll.201503334.

[11] J. Lv, Y. Song, L. Jiang, J. Wang, ACS Nano 2014, 8, 3152-3169.

[12] T. M. Schutzius, S. Jung, T. Maitra, P. Eberle, C. Antonini, C. Stamatopoulos, D. Poulikakos, Langmuir 2015, 31, 4807-4821.

[13] T. M. Schutzius, S. Jung, T. Maitra, G. Graeber, M. Köhme, D. Poulikakos, Nature 2015, 527, 82-85.

[14] Q. Zhang, M. He, J. Chen, J. Wang, Y. Song, L. Jiang, Chem. Commun. 2013, 49, 4516- 4518.

[15] A. Amirfazli, C. Antonini, in Non-Wettable Surfaces, RSC Soft Matter No. 5 (Eds: R. H. A. Ras and A. Marmur), Cambridge, UK 2017, pp. 319-346.

[16] S. Hoshian, V. Jokinen, K. Hjort, R. H. A. Ras, S. Franssila, ACS Appl. Mater. Interfaces 2015, 7, 15593-15599.

[17] J. Li, L. Li, X. Du, W. Feng, A. Welle, O. Trapp, M. Grunze, M. Hirtz, P. A. Levkin, Nano Lett. 2015, 15, 675-681.

[18] T. Sun, H. Tan, D. Han, Q. Fu, L. Jiang, Small 2005, 1, 959-963.

[19] M. Elsharkawy, T. M. Schutzius, C. M. Megaridis, Lab Chip 2014, 14, 1168-1175.

[20] A. B. D. Cassie, Discuss. Faraday Soc. 1948, 3, 11-16.

[21] H. Y. Erbil, A. L. Demirel, Y. Avc1, O. Mert, Science 2003, 299, 1377-1380.

[22] T. Sun, L. Feng, X. Gao, L. Jiang, Acc. Chem. Res. 2005, 38, 644-652.

[23] D. Quéré, Annu. Rev. Mater. Res. 2008, 38, 71-99.

[24] A. Lafuma, D. Quéré, Nat. Mater. 2003, 2, 457-460.

[25] G. Liu, L. Fu, A. V. Rode, V. S. J. Craig, Langmuir 2011, 27, 2595-2600. 


\section{WILEY-VCH}

[26] M. Reyssat, J. M. Yeomans, D. Quéré, EPL 2008, 81, 26006.

[27] P. Brunet, F. Lapierre, V. Thomy, Y. Coffinier, R. Boukherroub, Langmuir 2008, 24, 11203-11208.

[28] C. I. Park, H. E. Jeong, S. H. Lee, H. S. Cho, K. Y. Suh, J. Colloid Interface Sci. 2009, 336, 298-303.

[29] T. Verho, J. T. Korhonen, L. Sainiemi, V. Jokinen, C. Bower, K. Franze, S. Franssila, P. Andrew, O. Ikkala, R. H. A. Ras, Proc. Natl. Acad. Sci. 2012, 109, 10210-10213.

[30] Z. Cheng, H. Lai, N. Zhang, K. Sun, L. Jiang, J. Phys. Chem. C 2012, 116, 1879618802.

[31] C. Lee, Y. Nam, H. Lastakowski, J. I. Hur, S. Shin, A. L. Biance, C. Pirat, C. J. kim, C.Ybert, Soft Matter, 2015, 11, 4592-4599.

[32] E. Bormashenko, R. Pogreb, G. Whyman, M. Erlich, Langmuir 2007, 23, 12217-12221.

[33] Q. S. Zheng, Y. Yu, Z. H. Zhao, Langmuir 2005, 21, 12207-12212.

[34] E. Bormashenko, A. Musin, G. Whyman, M. Zinigrad, Langmuir 2012, 28, 3460-3464.

[35] P. R. Jones, X. Hao, E. R. Cruz-Chu, K. Rykaczewski, K. Nandy, T. M. Schutzius, K. K. Varanasi, C. M. Megaridis, J. H. Walther, P. Koumoutsakos, H. D. Espinosa, N. A. Patankar, Sci. Rep. 2015, 5, 12311.

[36] D. M. Lopes, S. M. M. Ramos, L. R. de Oliveira, J. C. M. Mombach, RSC Adv. 2013, 3, $24530-24534$.

[37] H. J. Butt, I. V. Roisman, M. Brinkmann, P. Papadopoulos, D. Vollmer, C. Semprebon, Curr. Opin. Colloid Interface Sci. 2014, 19, 343-354.

[38] J. T. Korhonen, T. Huhtamäki, O. Ikkala, R. H. A. Ras, Langmuir 2013, 29, 3858-3863.

[39] S. Srinivasan, G. H. Mckinley, R. E. Cohen, Langmuir 2011, 27, 13582-13589.

[40] J. V. I. Timonen, M. Latikka, O. Ikkala, R. H. A. Ras, Nat. Commun. 2013, 4, 2398.

[41] R. Massart, IEEE Trans. Magn. 1981, 17, 1247-1248.

[42] Y. Sahoo, A. Goodarzi, M. T. Swihart, T. Y. Ohulchanskyy, N. Kaur, E. P. Furlani, P. 
WILEY-VCH

N. Prasad, J. Phys. Chem. B 2005, 109, 3879-3885. 